\title{
CURVELET-BASED CHANGE DETECTION FOR MAN-MADE OBJECTS FROM SAR IMAGES
}

\author{
Andreas Schmitt, Birgit Wessel, Achim Roth
}

\author{
German Aerospace Center (DLR) \\ German Remote Sensing Data Center (DFD), D-82234 Wessling \\ Andreas.Schmitt@dlr.de,Birgit.Wessel@dlr.de,Achim.Roth@dlr.de
}

\begin{abstract}
In this article we present a technique for fast and robust change detection based on the curvelet transform. The curvelet transform is a two dimensional further development of the well-known wavelet transform that reconstructs the original image by ridge-like features, called ridgelets, in different scales, directions and positions. Geocoded SAR amplitude images from TerraSAR-X are compared by differentiating the coefficients of both images in the curvelet coefficient domain. Before the difference image is transformed back to the spatial domain, the influence of the single ridgelets on the resulting image can be manipulated to suppress noise and to intensify structures. Two examples were chosen to show the potential of this approach: a construction site in Germany and an open cast mining area in Chile. Our prototype version is able to compare time series without any interaction of an operator so that the implemented algorithms can easily be embedded into an automatic processing chain.
\end{abstract}

Index Terms - Synthetic aperture radar, Radar applications, Time series, Urban areas, Image enhancement

\section{INTRODUCTION}

The recent SAR systems like TerraSAR-X do not only allow a weather and illumination independent availability but also the capture of large coverages with high resolution. Hence, SAR sensors excel in the survey of changes in vulnerable structures, e.g. urban infrastructure. In the case of natural disasters a fast data acquisition becomes possible, but in order to provide a final product, i.e. damage map or accessibility map for roads, also "near realtime" a fully automatic data processing and interpretation is needed. A main problem for SAR image interpretation apart from the geometrical aspect is the high noise level caused by the speckle effect inter alia. The reduction of noise, e.g. by the multi-looking approach, often goes along with a loss of resolution. While structure preserving filters do not enhance fine structured areas, smoothening filters even blur the structures apparent in SAR data over urban areas. So, resolution and structure preserving filter algorithms are still a research topic. In this context alternative image representations excel: wavelets to separate point singularities or second generation wavelets to extract features of higher dimensions, e.g. lines or surfaces. For high resolution SAR data of urban areas, where not only streets or blocks but even single buildings are represented by lines of high (diplane) backscattering, the curvelet transform based on linear features seems to be most promising in this article. After a brief overview to related work, the method and its applications to SAR image enhancement as well as to change detection are presented (cf. Section 3). Then the results of two different test sites are shown and discussed (cf. Section 4) before the conclusion summarizes the main points and gives an outlook to future work.

\section{RELATED WORK}

The curvelet transform has originally been developed to describe an object with edges by a minimal number of coefficients in the continuous space. Much research work was done to transfer the definitions from the continuous to the discrete space and to accelerate the computation so that digital image processing becomes feasible [1]. Many applications in different scientific fields have been published so far, e.g. in geoand astrophysics, that are summarized in [2].

One wavelet-based approach for SAR image enhancement consists of a combination of the multi-scale representation and "standard" filtering techniques [3]. The advantage of second generation wavelets for despeckling has been examined for the Bandelet and the Contourlet transform [4]. In the remote sensing context only one publication [5] reports on denoising algorithms based an wavelets and curvelets after an initial total variation segmentation. Although its suitability for large scenes with different land cover types, we prefer a uniformely curvelet based method for purely urban applications to eliminate effects by the initial total variation segmentation.

Change detection in SAR images being a very difficult task has often been discussed in literature. One idea is to fuse several SAR images to a "superresolution" image [6], so that man-made objects, not captured in the digital elevation model 


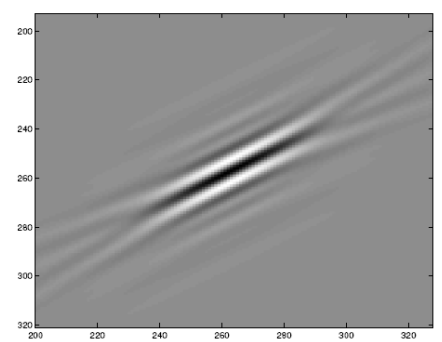

Fig. 1. Ridgelet in spatial domain [1]

used for the orthorectification, are classified by their diverse appearance in the single images. Unfortunately this method requires a great number of different SAR images. Another idea is to exploit the coherence (phase information) of an interferometric pair [7], which implies a relatively short repeatpass time to avoid additional incoherence caused by natural surfaces. Both are not suitable for a fast damage mapping after natural disasters.

\section{METHOD}

In this section the fundamentals of the approach are summarized. After a short introduction to the curvelet transform and its application to SAR data some image enhancement techniques are described. Finally the change detection based on the difference in the curvelet coefficient domain is presented.

\subsection{Curvelets}

The curvelet transform decomposes an image into a huge amount of ridgelets (see Fig. 1). Each ridgelet has a certain length, that corresponds to the scale while its width is determined by the law width $\approx$ length $^{2}$. In each scale a certain number of directions is distinguishable. While on the finest level (N4-neighbourhood), only horizontal and vertical ridgelets can be found, the number of possible directions increases with an increasing scale. Additionally the ridgelets can have different positions. The influence of each single ridgelet on the resulting image is weighted by a coefficient. The gray value of each resulting pixel is consequently determined by the summarized contributions of all ridgelets. To process radar data, which has a multiplicative character, the original gray values have to be scaled logarithmically to replace the multiplication by the sum. After the inverse curvelet transform the gray values are converted back to the original scale.

\subsection{Image Enhancement}

As every ridgelet coefficient in the coefficient domain corresponds to a ridge-like linear structure in the spatial domain it is possible to access directly the structures via the curvelet coefficients. For SAR applications four ways of manipulating the coefficients to reconstruct an enhanced image seem to be useful:

Scale One can choose the ridgelets to be preserved and those to be dropped by their belonging scale, e.g. setting all coefficients of the finest scale to zero means removing all small scale disturbances (cf. Fig. 2(b)). In a similar way the scales of interest can be narrowed down so that only the structures of interest remain in the resulting image. Over urban areas this procedure is very useful for edge detection.

Direction Alternatively the ridgelet can be chosen by its direction, i.e. linear structures of a certain direction are intensified while others are suppressed. The definition of a direction of interest in advance is not reasonable in most cases. But the directionality of the ridgelets can help to find the main axes of a city centre automatically because of the accumulation of strong ridgelets in the direction of the main roads. This effect can also be exploited to enhance orthogonal structures by taking into account the coefficients of the orthogonal direction.

Magnitude Independent of scale or direction the coefficients can be chosen only by their magnitude. The threshold can be found empirically or is determined by the image statistics. An adaptive threshold can be calculated for the whole image, for each scale or even for each direction. To show the effects of a variance dependent threshold the original SAR

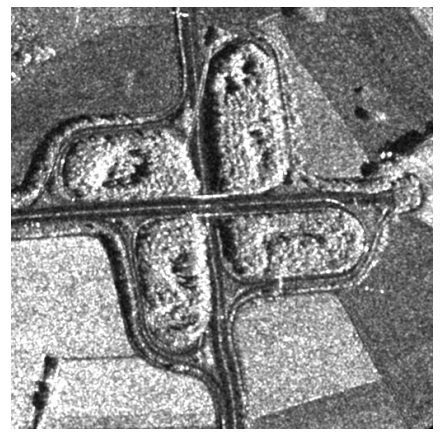

(a) Original amplitude image

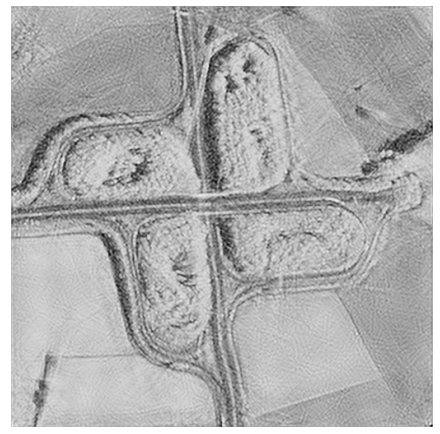

(c) Enhanced image with threshold

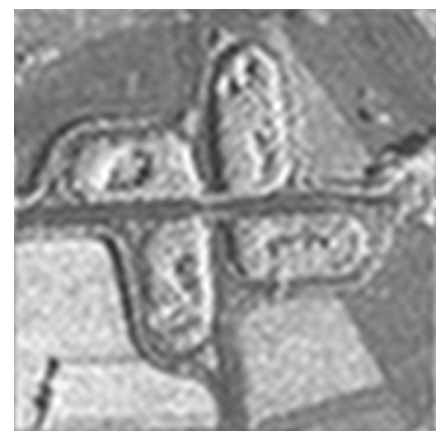

(b) Enhanced by dropping short lines

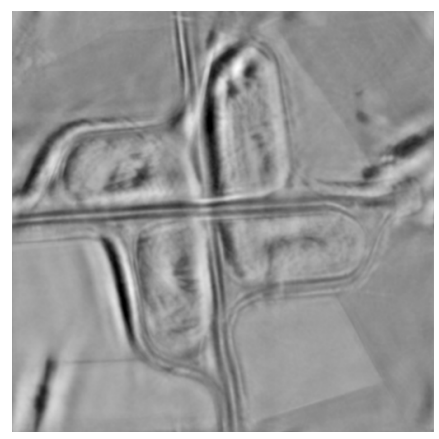

(d) Enhanced image with weighting
Fig. 2. Image enhancement with curvelets 
image (Fig. 2(a)) is reconstructed using only those coefficients that exceed the standard deviation of all magnitudes. Many artifacts, artificial linear structures, especially in originally smooth regions are obvious (Fig. 2(c)). Hence, the determination of a suitable threshold is a difficult task.

Enhancement by weighting instead of excluding some ridgelets from the reconstruction. Again, this weighting can depend on the scale or on the direction. In Fig. 2(d) a quadratic weighting of each single coefficient is applied. Low coefficients, whose magnitude is lower than one, are weighted down, stronger coefficients are even strengthened. Additionally the finest scale is completely set to zero. The resulting image is very smooth, but still contains the main structures. Unfortunately some stronger structures, like the shadow along the road in Fig. 2(d), often hide close-by lower structures. This weighting is more adequate for the enhancement of differences.

\subsection{Change Detection}

Input data for the curvelet-based change detection are two or more coregistered equally sized SAR amplitude images. For TerraSAR-X High Resolution Spotlight data the registration of the Enhanced Ellipsoid Corrected product type (EEC) turns out to be sufficient. To avoid geometrical distortions the images are acquired in exactly the same orbit. The developed prototype of a fully automatic routine cuts out overlapping areas of several images. If requested, the area of interest can be further restricted. All images are logarithmically scaled and transformed to the curvelet coefficient domain. The coefficient differences between two images are then weighted (magnitude square) and transformed back to the spatial domain. A colour coding to indicate areas that brightened up in green (positive values) and those that darkened in red (negative values) is performed and a transparency layer is added so that the result can be overlayed and directly compared with any other geocoded image product.

To enhance the difference of two images of different times a weighting is superior to a thresholding: First no structures get lost, because no ridgelets are set to zero, and secondly less artifacts are produced. As EEC products are used as input even SAR images with different acquisition geometries could be processed. But then the radar inherent distortions like layover and shadow, especially in urban areas, become visible in the detected changes.

\section{APPLICATIONS}

The described algorithm is now applied to two different test sites with completely different kinds of man-made objects: an open cast copper mine in Chile and a construction site in Germany. The data has been gathered by TerraSAR-X in the high resolution spotlight mode with $\mathrm{HH}$ polarisation and is processed as Enhanced Ellipsoid Corrected product.

\subsection{Open Cast Mining}

The colour composition in Fig. 3(a) shows three images over the Chuquicamata copper mine in Chile. The regions of high reflectivity caused by an extreme foreshortening effect are slightly smeared over during the geocoding step. One can note that something has changed around the deposit in the middle left of the image. The other images show the results of a curvelet-based change detection with weighted coefficients. In Fig. 3(b) series of red and green stripes along the mountain especially in the upper part are visible. In Fig. 3(c) these structures can be found in the lower part. Fig. 3(d), which depicts the changes between the first and the third image can be seen as sum of the two preceding images. The sequence of red (darkened) and green (brightened up) linear features is most remarkable. These changes indicate a systematic displacement of linear high backscatterers. An explanation can be found in the geometrical form of the mine: The deposit is built in terraces. If the deposition goes on, the edges of these terraces move and cause also a displacement of the bright lines in the SAR image, because of the reflector-like diplane backscattering at each stage. In contrast to the colour composition (Fig. 3(a)) the results are very smooth and show no single pixel changes in the surrounding (Fig. 3(b)-3(d)). So, this approach is capable to survey open cast mining activities even though it is not possible at the moment to determine the amount of soil moved.

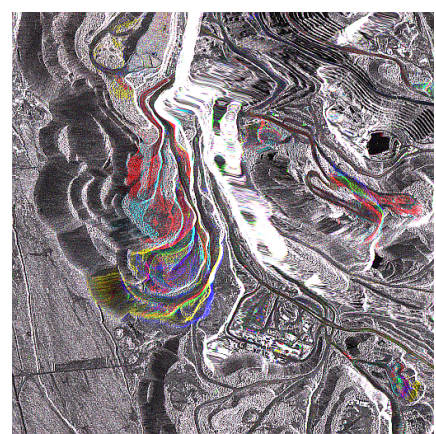

(a) Colour composit (1-2-3)

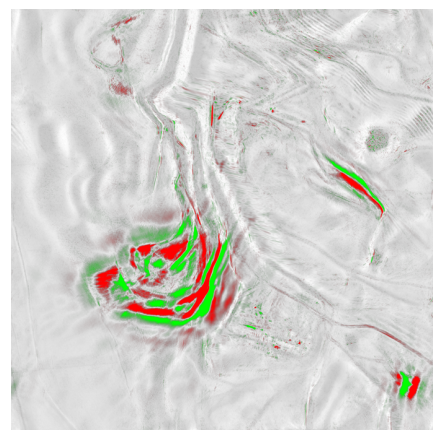

(c) Changes from 2 to 3

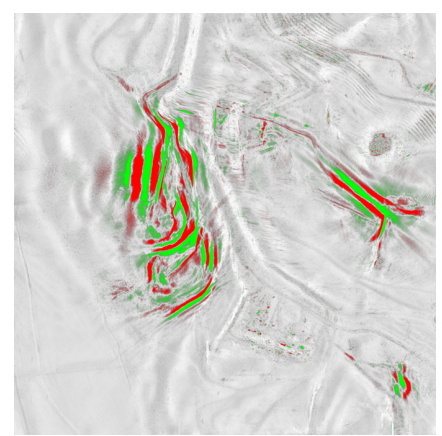

(b) Changes from 1 to 2

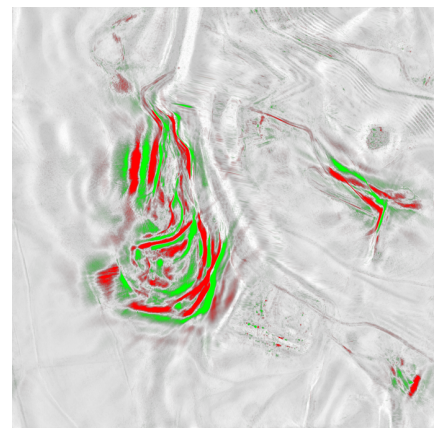

(d) Changes from 1 to 3
Fig. 3. Change Detection over open cast mining area, 1: 06.12.2008, 2: 08.01.2009, 3: 10.02.2009 


\subsection{Construction Site}

The construction site is situated near Ludwigshafen in Germany. Amidst agricultural land the surface is cleared and prepared for further construction (see Fig. 4(a)). Because of the plant's growth high differences in the surface roughness and therewith in the reflectivity are expected in the surrounding natural land cover. Having a look at the detected changes (Fig. 4(b), 4(c), 4(d)) in a series of four images, the future structure, probably the main streets, of the new enterprise zone become visible. But also in the surrounding some changes are mapped (see Fig. 4(d) at the right). So, it is not easy at all to decide, where the detected changes are man-made and where they are caused by the environmental conditions. With the first walls risen, stronger small scale changes should be detectable. Unfortunately the construction has not progressed during the winter months, so that the changes in the surrounding often were much higher than inside the construction site. Again, it is very helpful to have further information, e.g. land cover classification, to interpret correctly the results of the change detection. Especially for disaster applications it is essential to know whether the detected changes are situated in settlements or on cropland.

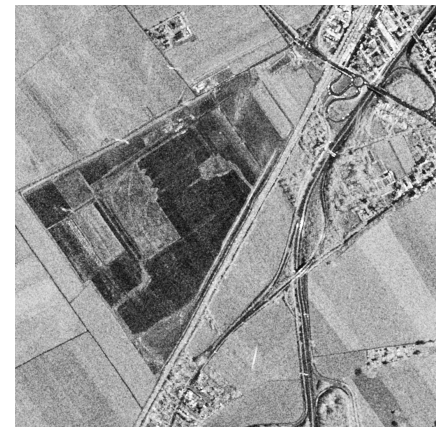

(a) Amplitude image

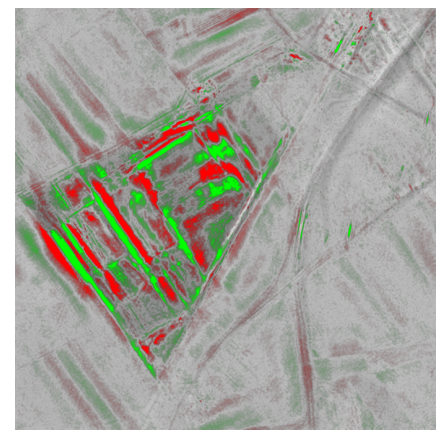

(c) Changes from 2 to 3

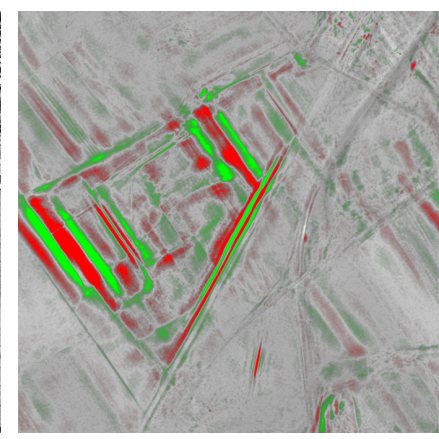

(b) Changes from 1 to 2

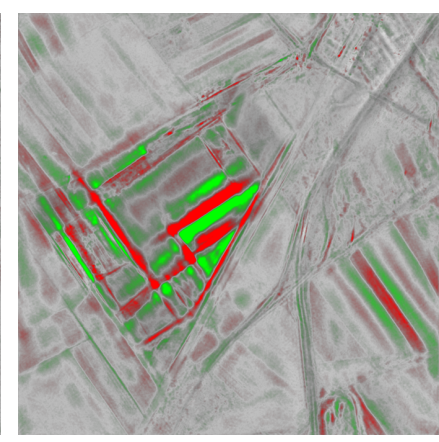

(d) Changes from 3 to 4
Fig. 4. Change detection over construction site, 1: 03.07.2008, 2: 23.11.2008, 3: 13.03.2009, 4: 18.05.2009

\section{CONCLUSION}

The presented curvelet-based approach for SAR image enhancement and change detection is a new and innovative method for SAR image analysis. On the one hand it is very flexibel because the operator can choose exactly what kind of structures are of interest and on the other hand it can also be designed as fully automatic routine, that can easily be embedded into a processing chain, e.g. for fast damage mapping. It is applicable to any kind of georeferenced SAR data as no phase information is needed. For change detection with TerraSAR-X Enhanced Ellipsoid Corrected products the geolocation is sufficient so that no further coregistration has to be done. Our future work will concentrate on the inclusion of multi-polarised data and phase information, e.g. via a coherence layer. Finally, we aim on a comparison of different SAR satellites.

\section{REFERENCES}

[1] E. J. Candès, L. Demanet, D. L. Donoho, and L. Ying, "Fast Discrete Curvelet Transforms," Multiscale Model. Simul., vol. 5, pp. 861-899, 2005.

[2] L. Demanet, “curvelet.org," http://www.curvelet.org, 2007, (accessed on 26 March 2009).

[3] S. M. Ali, M. Y. Javed, and N. S. Khattak, "WaveletBased Despeckling of Synthetic Aperture Radar Images Using Adaptive and Mean Filters," in Proceedings of World Academy of Science, Engineering and Technology, Venice (Italy), 2007, vol. 25, pp. 39-43.

[4] D. Gleich, M. Kseneman, and M. Datcu, "Despeckling of TerraSAR-X data using second generation wavelets," in ESA EUSC 2008: Image Information Mining, Frascati (Italy), 2008.

[5] J.R. Sveinsson and J.A. Benediktsson, "Combined wavelet and curvelet denoising of SAR images using TV segmentation," in Geoscience and Remote Sensing Symposium, IGARSS 2007, Barcelona (Spain), 2007, pp. 503-506.

[6] J.-S. Marcos, R. Romero, D. Carrasco, V. Moreno, J. L. Valero, and M. Lafitte, "Implementation of new SAR change detection methods: superresolution SAR change detector," in ESA-EUSC 2006: Image Information Mining for Security and Intelligence, Torrejon air base Madrid (Spain), 2006.

[7] P. Wright, T. Macklin, C. Willis, and T. Rye, "Coherent Change Detection with SAR," in European Radar Conference, EURAD 2005, Paris (France), 2005, pp. 17-20. 